\title{
ASSESSMENT OF THE EXPOSURE OF NURSES FROM EMERGENCY DEPARTMENIS TO AGGRESSIVE BEHAVIOR OF PATIENTS
}

\author{
Lukasz Czyzewski ${ }^{1,2}$, Katarzyna Rybak ${ }^{3}$ \\ ${ }^{1}$ Department of Nephrology Nursing, Medical University of Warsaw, Poland \\ ${ }^{2}$ Faculty of Health Sciences, Collegium Mazovia Innovative University, Siedlce, Poland \\ ${ }^{3}$ Student of Faculty of Health Sciences, Collegium Mazovia Innovative University, Siedlce, Poland
}

\begin{abstract}
INTRODUCTION: A large number of patients and their families waiting for help in ED, deteriorating health, high levels of stress and a recent sudden event, such as an accident, assault, life or health threaten the patient's anxiety, fear or fright that may result in irrational or violent behavior. These behaviors may be a threat to both the patient and the environment. This work aims to determine the exposure of nursing staff employed in ED for aggression on the part of patients.
\end{abstract}

METHODS: The research was carried out in the Masovian Province, in the Prague Hospital in Warsaw, in the Brodnowski Mazowieckie Hospital in Warsaw and in the Independent Public Health Care Team in Minsk Mazowiecki, from January to March 2018. The study was attended by 50 nurses working in ED.

RESULTS: According to the surveys carried out, nursing Staff in ED meets with the aggression of patients very often, ie at least once every few days - this is confirmed by as many as $60 \%$ of the respondents. For frequent contact with aggressive patients (ie on average once a week) indicates $28 \%$ of respondents, and the remaining $12 \%$ contact aggressive patients on average once a month. The most frequent type of aggression manifested by ED patients is verbal aggression $-68 \%$ of respondents contact it. $22 \%$ indicate physical aggression directed to nursing staff, and $10 \%$ for physical aggression but directed to objects.

CONCLUSIONS: In conclusion, our data indicate that: (1) Aggression of patients in ED is a very frequent phenomenon, and the level of high personnel exposure - aggression is recorded at least once every few days. There is no clear relationship between the increase in the number of aggressive patients on certain days of the week or the time of day, although there is a tendency to note such cases on weekends and at night; (2) The most common type of aggression undertaken by ED patients is verbal aggression manifested in insults, insults, shouting, threats and intimidation;

KEY WORDS: Emergency Department, nurse, aggression, burnout, violence

Disaster Emerg Med J 2018; 3(4): 111-118

\section{INTRODUCTION}

Aggressive behavior of patients in the Emergency Department $(E D)$ is an increasingly common phe- nomenon. A large number of patients and their families waiting for help in ED, deteriorating health, high levels of stress and a recent sudden event, such 
as an accident, assault, life or health threaten the patient's anxiety, fear or fright that may result in irrational or violent behavior. These behaviors may be a threat to both the patient and the environment. Acts of aggression are directed in such situations mostly towards medical personnel, including nurses taking care of the injured and performing various diagnostic and therapeutic procedures. Frequent contact of nursing staff with aggression in the workplace may cause a number of emotional and social effects and lead to loss of job satisfaction and finally to burnout. Due to the issues and high frequency of aggression in the work of ED nurses, this topic requires in-depth analysis.

This work aims to determine the exposure of nursing staff employed in ED for aggression on the part of patients.

\section{METHODS}

The research was carried out in the Masovian Province, in the Prague Hospital in Warsaw, in the Bródnowski Mazowieckie Hospital in Warsaw and in the Independent Public Health Care Team in Mińsk Mazowiecki, from January to March 2018. The study was attended by 50 nurses working in the ED. The respondents were informed about the purpose of the research and ensured full anonymity, and then asked to fill in the completed questionnaires. Before the start of the study, the permission of the management of the abovementioned institutions. The research tool used in this work was the original questionnaire consisting of 33 single-choice questions. All the questions were closed.

\section{RESULTS}

The study was attended by 50 nurses in the ED. The age of the subjects is between 22 and 56 years. The average age of the studied group is 37 years. The vast majority, i.e. $68 \%$ of the group were women, the remaining percentage of $32 \%$ were men. The group of respondents is characterized by diversity when it comes to seniority in the nurse's profession. The highest percentage of respondents is presented by people with work experience of 5-10 years - they constitute $38 \%$ of the group, $26 \%$ are people working in the profession above 15 years, 24\% work 11-15 years, and $12 \%$ less than 5 years.

According to the surveys carried out, nursing Staff in ED meets with the aggression of patients very often, i.e., at least once every few days. This is confirmed by as many as $60 \%$ of the respond- ents. For frequent contact with aggressive patients (i.e. on average once a week) indicates $28 \%$ of respondents, and the remaining $12 \%$ contact aggressive patients on average once a month.

The most frequent type of aggression manifested by ED patients is verbal aggression - $68 \%$ of respondents contact it. $22 \%$ indicate physical aggression directed to nursing staff, and 10\% for physical aggression but directed to objects.

The manifestation of verbal aggression is most often abusement and insults. It is indicated by $78 \%$ of respondents. In the second place, among the forms of verbal aggression, the subjects list threats and intimidation $(54 \%)$, shout $(54 \%)$, then raised the tone of voice $(46 \%)$, undermining competences and disregarding orders (40\%).

Among the forms of physical aggression manifested by the ED patients dominated by pushing - it was experienced by $64 \%$ of nursing staff and spitting - it was experienced by $62 \%$ of respondents. From other aggressive behaviors, the subjects mentioned: biting, kicking (26\%), beating (18\%), using an acute or other dangerous tools (10\%), strangling (4\%).

To the question: "Have you ever been beaten by a patient at ED?" the vast majority, i.e., $76 \%$ of the surveyed group, replied in the negative. The remaining $24 \%$ experienced such an event, with $16 \%$ experiencing beating once, and $8 \%$ several times.

When it comes to a physical attack with the use of a dangerous tool, the vast majority, i.e. $84 \%$ of respondents, denied that such situation occurred during their work, but $16 \%$ of nurses surveyed experienced such an event, while in $14 \%$ of such cases took place. one time, and $2 \%$ a few times.

On the question "Who more often behaves aggressively towards nursing staff?", 74\% of respondents state that aggressive behavior is more often taken by men, the remaining $26 \%$ of respondents indicate women.

Regarding the age of patients who show aggression towards nursing staff, it turns out that in the majority, i.e. $66 \%$, they are people between 19 and 33 years of age. The average age is indicated by $24 \%$ of respondents, and very young people (up to 18 years of age) indicate $10 \%$ of the examined group.

Aggression in ED - as indicated by $74 \%$ of the studied group - is most often demonstrated by patients under the influence of alcohol. The second group of patients - in terms of the frequency of in- 
toxication with drugs or afterburners - is indicated by $20 \%$ of respondents. The remaining $6 \%$ mention patients in acute psychosis.

According to $76 \%$ of respondents, the aggression of patients in ED branches is not related to their disease. Only $24 \%$ of respondents indicate such a relationship.

The subjects were to determine on what days the aggressive patients can most often be found in ED. Half of the study group (50\%) reported that it did not matter, but $38 \%$ indicated weekends as the most common days in which aggressive patients occur. The remaining $12 \%$ of the group gave weekdays.

Also when it comes to the time of the day in which aggressive patients get most often on the $E D$, more than half of the respondents (54\% of the group) indicated that this is not important, 30\% reported that such patients most often occur at night, $12 \%$ reported that in the evening and $4 \%$ during the day.

The respondents were asked whether, despite the phenomenon of aggression, patients feel safe while working at ED. More than half of the respondents, i.e. $54 \%$, answered that definitely yes, $34 \%$ admitted that they feel moderately safe, while $12 \%$ admitted that they probably do not feel safe in their work.

When asked what you are most afraid of aggressive patients, the highest percentage of people, i.e. $44 \%$ of the group indicated injury and infection, e.g. HIV or HCV, a large part of the group, i.e. $38 \%$, is also afraid of a physical attack, e.g. the patient. $18 \%$ of respondents state that they are afraid of insults and $4 \%$ of humiliation or undermining competences.

In the case when the patient exhibits verbal aggression, the majority of respondents, i.e. $32 \%$ of the group, try to calm the patient, talk to him in a gentle tone, explain the purpose of the activities performed. $26 \%$ say that in such a situation, it usually ignores the aggressive behavior of the patient without paying attention to insults or insults. The remaining $18 \%$ take a firm stance and threaten the consequences that may occur.

In the case of physical aggression, more than half of the respondents, i.e. $54 \%$, call the police, $32 \%$ use means of direct coercion, for example, immobilization, and $14 \%$ try to calm the patient, calm down, speaking in a gentle tone.

On the question "Did you happen to call the police for an aggressive patient at ED?", the vast major- ity, as many as $94 \%$ of the respondents were forced to do so many times, the remaining $6 \%$ were calling the police once. There was not a single person in the surveyed group who would never face the necessity of summoning the police during their work in ED.

The vast majority of respondents, i.e. $88 \%$ of the total, were also forced to repeatedly apply coercive measures, the remaining $12 \%$ faced this situation once. There was not a single person who would not have had a situation in which it was necessary to use direct coercion.

The most frequently used means of direct coercion was immobilization using belts, handles, etc. This type was used by as much as $70 \%$ of respondents. $22 \%$ used holding, i.e., temporary immobilization of the patient using physical force, and $8 \%$ administered sedation without the consent of the patient.

On the question of whether experiencing aggression from patients results in lower satisfaction and job satisfaction in ED, 36\% of respondents answered that they did not, and $24 \%$ said that it does not affect their job satisfaction in any way. However, $28 \%$ of the group declares that in their case a decrease in satisfaction with work definitely declined, and $12 \%$ confirm a certain decrease in job satisfaction, but to a small extent.

Most of the respondents, i.e. $86 \%$ of the group, like their work in ED, $46 \%$ definitely confirm it, and $40 \%$ say that it is rather yes. Due to the phenomenon of aggression among patients, $8 \%$ of respondents like their work on average, $4 \%$ do not like what they do, and $2 \%$ definitely do not like working in ED.

Respondents were also asked if they had avoided and withdrawn from their duties due to a verbal or physical assault on the part of the patient. Most of the respondents, i.e. $74 \%$ of the group answered the question with negative, while $24 \%$ admitted that they occasionally had to withdraw from their official duties, and $2 \%$ confirmed that this situation happened to them often.

The vast majority of respondents, ie $66 \%$ of the group never thought about giving up their profession because of aggression on the part of patients. $24 \%$ of respondents admitted that they did it only once, while $10 \%$ confirmed that they had repeatedly thought about giving up their profession.

$66 \%$ of respondents never thought that due to patients' aggression, they would move to another ward, $16 \%$ of the group thought about it once. 
$10 \%$ of respondents declare that they think about it and plan to move to another branch, the remaining $8 \%$ confirms that they think about it many times.

More than a half of respondents, i.e. 54\%, think that aggression of patients is not a big problem in the work of nursing staff at ED, but a large group, ie $34 \%$, definitely considers this a significant problem in their work.

The question "Is there a need to implement additional measures to protect nursing staff at ED?" most respondents, i.e. $56 \%$, say that there is no need because the current measures are sufficient. 34\% have no opinion on the subject, and the remaining $10 \%$ say that additional measures are needed.

Most of the respondents, i.e. $58 \%$, also think that there is no need for nursing staff to participate in training, training or meetings with a psychologist improving skills in dealing with patients' aggression. $24 \%$ would rather see such a need, and $18 \%$ strongly indicate the need to organize such training.

As results from the research, after the incident in which an attack of aggression on the part of the patient occurred, not one of the respondents received help aimed at releasing negative emotions or coping with a difficult situation. The lack of such intervention was declared by $56 \%$ of respondents, as the remaining $44 \%$ did not experience an aggression attack from the patient.

Most of the respondents, i.e. $60 \%$, think that there is no need to implement interventions aimed at helping in coping with difficult emotions after a patient's attack, $22 \%$ would rather like this kind of help, and $18 \%$ would definitely confirm this need.

\section{DISCUSSION}

Aggression is an action oriented towards the outside or towards one's own person, undertaken in order to cause physical or psychological damage. It is intentional behavior that poses a threat or causes damage to the physical, mental and social well-being of others, leading to the loss of valued values [1]. In general terms, the concept of aggression refers to any actions that may be caused by the presence of such factors as fear, frustration, the desire to provoke fear or frustration in other people, the tendency to enforce other views. It can be a manifestation of the will to power and control others.

Aggressive behavior may be manifested in the form of physical and psychological aggression. Physical aggression is demonstrated by a physical attack (e.g, impact, pushing, jerking, kicking, strangling), which may result in the victim being injured, injured or damaged by objects in the environment [2]. Physical aggression can be directed against each other (self-harm, e.g. self-mutilation, suicide), against other people (nurses, doctors, other patients) or against objects (medical equipment, ambulance) [3]. In the case of psychological aggression, the indicators of such behavior are, for example, screaming, raised voice, verbal threats, intimidation, blackmail, use of profanity, insults, as well as offensive gestures, dangerous postures, fists, spitting, insults [2].

Clinical Classification of Aggression takes into account the division into aggressive behaviors in the affection - it is defensive aggression, "hot-blooded" aggression and aggressive behavior is taken to control the situation - it is instrumental and preventive ("cold-blooded" aggression) aggression. According to this division, impulsive aggression means uncontrolled reaction to verbal or physical aggression directed against the person under the influence of emotions. Instrumental aggression, on the other hand, is intentional behavior, undertaken in order to intimidate, gain dominance and control over the environment [1].

Considering the severity of aggressive behavior and its harmfulness to the environment, three groups characterizing aggressive patients can be selected. The first of them is created by patients who undertake the least threatening behaviors - these behaviors are defined as physical and verbal arousal. The second group includes patients using verbal aggression, for example, curses, insults, profanity, spitting and active aggression such as destruction of objects, biting, jerking [4]. The third group are patients with the highest degree of risk manifesting aggression causing bodily injury, eg strangling, mutilation, arson, attempted homicide, etc. [1].

\section{Causes of aggression in patients treated in the ED}

Aggressive behavior of patients who are sent to ED may be caused by a variety of factors, the most common of which are: mental disorders (psychosis, manic states, anxiety), personality disorders, withdrawal and poisoning syndromes (eg alcohol, amphetamine, cocaine), organic changes in the central nervous system, epilepsy, somatic diseases (eg thyroid dysfunction, hypoglycaemia, acidosis) [5]. They can also be caused by psychological or situational factors and constitute a strong reaction to stress (eg accident, assault, rape or other traumatic events) 
[6]. The risk of aggression is greater in people who have experienced physical violence in the past, were sexually abused, and were not accepted by the environment [2]. Aggression manifested by patients in ED may also be caused by the organization of work in the ward and the attitude and form of communication of nurses or other members of the medical team, e.g. low quality of the therapeutic relationship, lack of experience and appropriate qualifications of personnel or errors in communication with patients [4].

\section{Mental illness}

Aggressive behaviors are often the result of reduced impulse control caused by the presence of mental illness. Clinical experience indicates that mentally ill people are more likely to display aggression and commit illegal and sometimes violent acts. About $10 \%$ of chronically mentally ill patients exhibit aggressive behavior before they enter a psychiatric hospital. It has been proved that patients in a psychotic state, experiencing production symptoms in the form of imperative hallucinations, become the perpetrators of very serious violent acts, including even murders. In most cases, they do not take medicine in the period before they are committed.

The cause of aggression can be both anxieties, a sense of threat as well as psychotic experiences of the patient. Anxiety most often accompanies psychoses and disorders of panic disorder and is associated not only with psychological discomfort, a feeling of threat and fear, but also the occurrence of somatic symptoms in the form of tachycardia, dizziness, nausea, feelings of suffocation, cold and hot flushes, numbness, sweating, etc. In panic disorders, fear of impending death, infarction or loss of control is typical. In psychotic disorders, the basis of anxiety are production symptoms, i.e. delusions or hallucinations. The treatment of patients with panic disorder relies on sedation, administration of anti-anxiety agents, e.g. benzodiazepines, and in psychotic states also neuroleptics. It is important that anxiety disorders properly differentiate with somatic states in which anxiety may be secondary (e.g. in respiratory failure, myocardial infarction, etc.) [7].

Aggression can also occur in states of exacerbation of schizophrenia, when the predominant experiences are hostility, distrust, suspicion and dislike towards other people, resulting from fear of their intentions.

\section{Addiction to alcohol, drugs}

Aggression displayed by patients is not always the result of fear, pain or mental illness, it is repeatedly the result of an earlier consumption of alcohol or other psychoactive substances, eg drugs or afterburners [8]. A large proportion of patients who are sent to $E D$ are under the influence of alcohol. Alcohol intoxication very often leads to poisoning, complications caused by its long-term abuse and is the cause of injuries and accidents [9]. Data from the literature indicate that patients with an injury who are under the influence of alcohol and/or drugs account for $40 \%$ of the victims who receive help in ED. This percentage is systematically increasing every year [8].

Aggression of patients under the influence of psychoactive substances can occur in both intoxication states and withdrawal syndromes associated with alcohol withdrawal, amphetamine, cocaine (especially at the beginning of abuse and secondary to delusional content in the late period of addiction), hypnotics and anxiolytics (benzodiazepines, barbiturates) narcotic analgesics, steroids (prednisone, cortisone, anabolic steroids) [5].

The relationship between drinking alcohol and aggressive behavior is high. After drinking alcohol, the psychopharmacological effect of intoxication with alcohol appears; in the disinhibition of the individual, distortion of its cognitive-perceptual processes and the occurrence of attention deficits. These processes, combined with situational factors associated with drinking, trigger aggressive behavior. The intensity of aggression depends then on the basic, basic level of aggression of a given person. Aggressive behavior is also recognized as the effect of self-control as a result of drinking alcohol. Consumption of alcohol undoubtedly lowers the threshold of appearance of aggressive behavior, which means that even a small reason is enough to start an aggressive reaction in the unit [10].

The use of a psychoactive substance can be suspected when the patient is uncritical, has anxiety, anxiety, and a changing mood. When morphine or its derivatives have been admitted, one can additionally notice narrowed pupils, a glazed look, pale skin, and if it is discontinued, then the pupillary dilation, tearing, sneezing, increased sweating, nausea, and yawning will be observed. The reception of amphetamines indicates psychomotor agitation, mydriasis, fast breathing, insomnia, after taking cocaine there are also pimples on the red nose. Psychotic symptoms may also appear in any 
psychoactive substance. In the event of a sudden withdrawal of benzodiazepines, the following may occur agitation, anxiety, anxiety, increased sweating, nausea, vomiting, tremor or muscle spasms, and even epileptic seizures [6].

\section{Neurological disorders, CNS damage}

Aggressive behaviors are often observed in patients with organic changes in the central nervous system. Such changes may lead to emotional and behavioral disorders and result in strong arousal. Aggression may be manifested by patients in acute states with impaired consciousness (e.g ., delirium of various etiologies, clouding, drowsiness) as well as patients with dysphoria without consciousness disorders in chronic neurological diseases (e.g., in Parkinson's disease, Huntington's disease, Wilson's disease, multiple sclerosis), in lupus nodules), in metabolic disorders (e.g., thyroid disease, porphyria, hypoglycaemia, acidosis, vitamin B12 deficiencies), in dementia (Alzheimer's type, vascular, mixed).

Neuroimaging brain tests carried out in aggressive and antisocial people reveal the presence of subtle changes in the volume of the gray matter and white prefrontal and temporal cortex. These changes are probably the result of neurodevelopmental disorders, which means that they are innate. Scientific knowledge about the role of the limbic system and the hypothalamus in the manifestation of aggression is based mainly on the study of the correlation of behavior and the occurrence of aggressiveness in patients with neurological disorders. Aggressiveness often accompanies diseases such as temporal epilepsy, temporal lobe sclerosis, tumors in these regions and in other areas of the limbic system and hypothalamus [11].

\section{Injuries, injuries, somatic diseases}

The tendency towards aggressive behavior increases in the case of some diseases or somatic states, in particular: endocrine diseases (mainly thyroid dysfunction), metabolic (eg hypoglycaemia, acidosis, hypoxia, vitamin deficiencies, e.g. B12, porphyria, metabolic disorders in the course of kidney failure, liver), neurological (multiple sclerosis, visceral lupus, meningitis, stroke), head injuries, brain tumors [6].

Violent, aggravated arousal leading to aggressive behaviors may also be caused by disturbances of consciousness in the course of hypoxia due to breathing disorders or serious cardiac disorders. Cardiac conditions such as myocardial infarction or arrhythmia cause anxiety, which in turn may trigger aggression directed at, for example, nurses who give help. Anxiety also coincides with episodes of hypoglycemia and pheochromocytoma. Repeatedly in situations of serious somatic health disorder, a delirium syndrome that is an impairment of consciousness develops. Such a condition may occur, for example, in hepatic encephalopathy or disturbances in water and electrolyte management [7]. Also in some infections, poisoning with mercury and steroid treatment, mainly anabolic - mania or hypomania may occur, which is usually accompanied by a high level of irritability and aggressiveness [5].

\section{Actions aimed at reducing aggression in patients}

The nursing staff in ED should know the rules of dealing with an aggressive and aggressive patient so that activities undertaken by the entire medical team are not mutually exclusive and do not cause chaos. Agitation of a patient caused by a serious physical condition constituting a real threat to life is generally short-lived - it results from a sudden deterioration of health and results in impulsive behavior, which, however, is chaotic and does not lead to harm to another person. The patient in this condition should be pacified and the diagnostic and therapeutic actions should be taken towards normalizing the somatic state. If the patient is aroused or aggressive, and his/her physical state is not life-threatening, first of all, one should take care of the safety of their own, staff and other patients. The basic safety rules are: avoiding being with an aggressive patient in a closed room, not reaching the distance of arms extended, quickly informing other people about the danger and calling for help, restricting access to sharp or other dangerous objects, eg glass dishes, cutlery, syringes, scissors, ashtrays, as well as access to electricity, boiling water, etc. [6].

In a situation where there is a real possibility of aggression attack on the part of the patient, it should be in a non-confrontational way to show him his advantage and readiness to use it, he must not react with fear. It is necessary to show the limits of unacceptable behaviors, at the level of verbal aggression. It is helpful to observe your own experiences because often before the appearance of aggression in the patient, we feel unspecified anxi- 
ety, which can be a warning signal anticipating the reactions of an aggressive person [7].

The ability to handle an aggressive patient, the ability to predict aggressive behavior and the use of appropriate procedures and activities is associated with the experience of medical personnel in such situations. Staff should avoid actions and behaviors that could be a provocation to aggression. The patient should be treated with respect, give him information and answers simple and straightforward, show interest, create conditions allowing free expression of emotions, provide a sense of security, friendly atmosphere, eliminate factors that increase tension, do not interfere in an exaggerated manner and excessively embarrassing, reverse attention from the cause of the conflict. Nursing staff showing understanding and kindness, empathy, and trust are able to cope much better with the aggressive behavior of the patient. Personnel working in hospital emergency departments should look after the sick in such a way that their possible aggressive behaviors are properly identified, effectively minimized and the proper procedures implemented [4].

\section{Means of direct coercion}

If the aggressive behavior of the patient begins to exceed the physical limits, it is necessary to take decisive action. In the case of the patient's lack of reaction to the orders to stop aggression, it is necessary to apply direct coercive measures [7].

Direct coercive measures include holding, compulsory medication, immobilization and isolation [12].

Clinical experience shows that the most commonly used means of direct coercion is immobilization and holding. However, for any type of direct coercion, it is necessary to consider which one will be the least onerous for the patient himself. The fact of applying direct coercion, as well as information about the patient's prior warning about the necessity of its use, must be recorded in the medical documentation.

Direct coercive measures are used when the patient experiences aggressive behavior, and other ways of calming him have failed and no other way to control aggression is possible. The implementation of these measures enables further performance of diagnostic and therapeutic activities for the patient, as well as protects the environment and the patient against aggression on his part. They can only be used until the reasons for protection has subsided [6]. During the entire period of direct coercion, it is important to ensure comfort, safety, and intimacy to the patient [13].

\section{Legal protection of nurses}

The rights of medical personnel, including nurses, are protected by the applicable legal system, including criminal and civil law norms. From the perspective of criminal protection, it is important that the nurse, during and in connection with the performance of activities consisting in the provision of health services, benefits from the protection provided for public officials on the basis of art. 11 para. 2 of the Act on Nurse and Midwife Professions [14]. Due to the fact that the nurse is entitled to legal protection as a public official, the patient may be prosecuted under Art. 222 k.k., 223 k.k. and 226 k.k. These provisions provide for successive liability for violation of bodily integrity, active assault, and insult [15].

In addition to criminal sanctions, a patient who engages in aggressive behavior towards medical personnel may also incur civil liability for violation of personal rights.

A nurse who has experienced aggression on the part of the patient may demand their rights and make a civil claim, file a crime notice or file a private indictment. In order to pursue claims, the aggressive behavior of the patient should be recorded in the medical documentation, taking into account the circumstances of the occurrence, the manner of the perpetrator's action, determining the place and time of the incident and giving witnesses. It is also necessary to draw up a staff memo, notify superiors about the situation and indicate any injuries documented by the medical prescription. These actions should be performed immediately after the occurrence of the incident [15].

\section{Threats resulting from frequent aggression at work}

Aggression experienced in work is one of the most serious factors changing the workplace into a threatening and even repulsive place [16]. According to Nolan et al. [17] experiencing aggression significantly correlates with the lack of job satisfaction, because it causes a decrease in involvement in professional duties, and thus leads to a deterioration of contact with patients or a team.

The effects of experiencing aggression can be varied - from physical injuries to psychological reactions and injuries. A natural response to an attack on the part of the patient is feeling fear, helplessness, an- 
ger, which emotions can trigger anxiety attitude and secondary lead to avoidance and withdrawal from their professional duties, cause a decrease in work efficiency, or even willingness to resign from further profession or cause aggression against the attacking patient [17]. In addition, an event with an aggressive patient causes negative associations, which may also affect the attitude of the staff towards other patients and, as a result, interfere with the treatment process [12]. Finally, as a result of contact with an aggressive patient (especially if a physical attack has occurred), a post-traumatic stress disorder (PTSD) may develop causing serious dysfunctions and difficulties in everyday functioning [17].

A serious effect of experiencing aggression in everyday work is the development of burnout syndrome. Attempts to self-cope with the aggression of patients usually involve distancing oneself from the problem, minimizing the effects of aggression experienced, rationalizing, intellectualizing, projecting, undertaking altruistic and missionary actions [1]. The literature emphasizes the role of paying attention to the situation of aggression and training of medical personnel in recognizing symptoms of aggressive behavior and coping with them at work. It is extremely important to make the medical staff aware that some of his behaviors, such as the lack of assertiveness, the lack of setting boundaries in contact, are conducive to aggression by patients. Therefore, apart from the employee protection program, it is very important to conduct training for personnel, taking into account such issues as crisis intervention, techniques for identifying and dealing with aggression, self-defense, and incapacitating techniques, the correctness of reporting and recording incidents of aggression [17].

In conclusion, our data indicate that: (1) Aggression of patients in ED is a very frequent phenomenon, and the level of high personnel exposure - aggression is recorded at least once every few days. There is no clear relationship between the increase in the number of aggressive patients on certain days of the week or the time of day, although there is a tendency to note such cases on weekends and at night; (2) The most common type of aggression undertaken by ED patients is verbal aggression manifested in insults, insults, shouting, threats and intimidation; (3) Aggressive patients are usually people between 19 and 30 years of age, under the influence of alcohol or drugs, men; (4) In the case of verbal aggression, nursing staff usually calms patients or ignores their behavior. In the case of physical aggression, the most frequently intervened intervention is to call the police or apply direct coercion measures - before the intervention of these types of interventions, the respondents would be repeated many times. The most commonly used means of direct coercion is immobilization.

\section{REFERENCES}

1. Mosiołek A, Koweszko T, Gierus J. Aggression in psychiatric institutions — an attempt to define the problem. Psychiatry. 2014; 11: 88-91.

2. Frydrysiak K, Grześkowiak M, Podlewski R. Patient aggression as a threat to the work of emergency teams. Anaesthesiology \& Rescue Medicine. 2014; 8: 381-391.

3. Sariusz-Skąpska M. Forms of patients' violent behaviour vs. occupational burnout in the staff of psychiatric services. Advances in Psychiatry and Neurology. 2005; 14: 87-91.

4. Markiewicz R. Aggressive behavior of patients towards nursing staff employed in psychiatric wards. Current Problems of Psychiatry. 2012; 13: 93-97.

5. Rzewuska M. Pharmacological treatment for aggressive patients with psychiatrie disorders. Pharmacotherapy in Psychiatry and Neurology. 2004; 3: 293-323.

6. Rymaszewska J. The treatment of an aggressive and agitated patient. Psychiatry in Clinical Practice. 2008; 1: 74-78.

7. Furgał $M$, Heitzman J. Sudden states in psychiatry [w:] J. Heitzman (red.) Psychiatry. Manual for medical studies, PZWL. 2007: 246-247.

8. Wnukowski K, KopańskiZ, Brukwicka I, et al. The dangers of medical rescue work-selected issues. Journal of Clinical Healthcare. 2015; 3: 10-16.

9. Rudnicka-Drożak E, Misztal-Okońska P, Młynarska M. Opinions of the hospital emergency department workers on the subject of providing medical assistance to patients with alcohol poisoning - a preliminary report. Problems of Hygiene and Epidemiology. 2013; 94: 577-582.

10. Kosińska-Dec K, Jelonkiewicz I. Alcohol drinking and jnterpersonal aggression among young adults. Alcoholism and Drug Addiction. 2000; 13: 223-233.

11. Vetulani J. Neurochemistry of impulsiveness and aggression. Psychiatr Pol. 2013; 47(1): 103-115, indexed in Pubmed: 23888748.

12. Kliś A. Prophylaxis of patients' aggression and violence in dialysis centres. Forum Nefrologiczne. 2011; 4: 177-182.

13. Bieda M. Behavior towards people with mental illness and mental disorder manifested in the practice of medical rescuer. Krakow Academy Andrzej Frycz Modrzewski, Krakow 2015.

14. Dz.U.2018.0.123 Act of July 15, 2011 on nurse and midwife professions.

15. Augustynowicz A, Czerw A, Wrześniewska-Wal I, et al. Legal protection of the nurse / midwife in case of aggressive behavior of patients in therapeutic entities. Journal of Education, Health and Sport. 2015; 5: 259-268.

16. Anczewska M. Stress and burnout in psychiatric health care workers. Institute of Psychiatry and Neurology, Warszawa 2006.

17. Lickiewicz J, Piątek J. Nurses experiencing aggression at workplace. Art of Treatment. 2014; 3-4: 11-22. 